\title{
The impact of demographic, clinical, genetic, and imaging variables on tau PET status
}

\author{
Rik Ossenkoppele ${ }^{1,2}$ (1) Antoine Leuzy ${ }^{1} \cdot$ Hanna Cho $^{3} \cdot$ Carole H. Sudre ${ }^{4,5,6} \cdot$ Olof Strandberg $^{1} \cdot$ Ruben Smith $^{1}$. \\ Sebastian Palmqvist ${ }^{1} \cdot$ Niklas Mattsson-Carlgren $^{1,7,8} \cdot$ Tomas Olsson $^{9} \cdot$ Jonas Jögi $^{10}$ • Erik Stormrud ${ }^{1,11}$. \\ Young Hoon Ryu ${ }^{12} \cdot$ Jae Yong Choi ${ }^{12,13}$. for the Alzheimer's Disease Neuroimaging Initiative $\cdot$ for the PREVENT-AD \\ research group - Adam L. Boxer ${ }^{14}$. Maria L. Gorno-Tempini ${ }^{14}$ • Bruce L. Miller ${ }^{14}$. David Soleimani-Meigooni ${ }^{14}$. \\ Leonardo laccarino ${ }^{14} \cdot$ Renaud La Joie ${ }^{14}$. Edilio Borroni ${ }^{15} \cdot$ Gregory Klein $^{15}$ - Michael J. Pontecorvo ${ }^{16}$. \\ Michael D. Devous $\mathrm{Sr}^{16}$. Sylvia Villeneuve ${ }^{17}$. Chul Hyoung Lyoo ${ }^{3}$. Gil D. Rabinovici ${ }^{15,18,19} \cdot$ Oskar Hansson ${ }^{1,11}$
}

Received: 24 September 2020 / Accepted: 27 October 2020 / Published online: 19 November 2020

(C) The Author(s) 2020

\begin{abstract}
Purpose A substantial proportion of amyloid- $\beta(\mathrm{A} \beta)+$ patients with clinically diagnosed Alzheimer's disease (AD) dementia and mild cognitive impairment (MCI) are tau PET-negative, while some clinically diagnosed non-AD neurodegenerative disorder (non-AD) patients or cognitively unimpaired (CU) subjects are tau PET-positive. We investigated which demographic, clinical, genetic, and imaging variables contributed to tau PET status.

Methods We included 2338 participants (430 A $\beta+$ AD dementia, $381 \mathrm{~A} \beta+\mathrm{MCI}, 370$ non-AD, and $1157 \mathrm{CU}$ ) who underwent $\left[{ }^{18} \mathrm{~F}\right]$ flortaucipir $(n=1944)$ or $\left[{ }^{18} \mathrm{~F}\right] \mathrm{RO} 948(n=719)$ PET. Tau PET positivity was determined in the entorhinal cortex, temporal meta-ROI, and Braak V-VI regions using previously established cutoffs. We performed bivariate binary logistic regression models with tau PET status (positive/negative) as dependent variable and age, sex, APOE\&4, A $\beta$ status (only in CU and nonAD analyses), MMSE, global white matter hyperintensities (WMH), and AD-signature cortical thickness as predictors. Additionally, we performed multivariable binary logistic regression models to account for all other predictors in the same model. Results Tau PET positivity in the temporal meta-ROI was $88.6 \%$ for AD dementia, $46.5 \%$ for MCI, $9.5 \%$ for non-AD, and $6.1 \%$ for CU. Among A $\beta+$ participants with AD dementia and MCI, lower age, MMSE score, and AD-signature cortical thickness showed the strongest associations with tau PET positivity. In non-AD and CU participants, presence of A $\beta$ was the strongest predictor of a positive tau PET scan.
\end{abstract}

Data used in preparation of this article were obtained from the Alzheimer's Disease Neuroimaging Initiative (ADNI) database (adni.loni.usc.edu). As such, the investigators within the ADNI contributed to the design and implementation of ADNI and/or provided data but did not participate in analysis or writing of this report. A complete listing of ADNI investigators can be found at: http://adni.loni.usc.edu/wpcontent/uploads/how to_apply/ADNI_Acknowledgement_List.pdf

Data used in preparation of this article were obtained from the Presymptomatic Evaluation of Novel or Experimental Treatments for Alzheimer's Disease (PREVENT-AD) program (https://douglas. research.mcgill.ca/stop-ad-centre). A complete listing of PREVENT-AD Research Group can be found in the PREVENT-AD database: https:// preventad.loris.ca/acknowledgements/acknowledgements.php?date= [2020-09-08]†.

This article is part of the Topical Collection on Neurology

Rik Ossenkoppele

r.ossenkoppele@amsterdamumc.nl
Oskar Hansson

oskar.hansson@med.lu.se

Extended author information available on the last page of the article 
Conclusion We identified several demographic, clinical, and neurobiological factors that are important to explain the variance in tau PET retention observed across the AD pathological continuum, non-AD neurodegenerative disorders, and cognitively unimpaired persons.

Keywords PET $\cdot$ Tau $\cdot \mathrm{A} \beta \cdot$ Alzheimer's disease $\cdot$ MCI $\cdot$ Dementia

\section{Introduction}

Tau accumulation is a key neuropathological feature of Alzheimer's disease (AD) and is closely linked to synaptic loss, neurodegeneration, and cognitive deficits [1]. The advent of positron emission tomography (PET) ligands with high affinity for the tau aggregates formed in AD now enables the visualization and quantification of tau pathology in vivo [2]. Recently, one of these tau PET tracers $\left(\left[{ }^{18} \mathrm{~F}\right]\right.$ flortaucipir $)$ was approved by the US Food and Drug Administration to support the diagnostic process in patients with suspected AD dementia [3]. This is an important step towards the clinical application of tau PET.

However, several hurdles need to be overcome in order to accelerate the transition of tau PET from an investigational technique to a diagnostic biomarker. One of these challenges is the observation of negative tau PET scans in individuals suspected of having symptomatic AD [4-7] or, conversely, positive tau PET scans in individuals suspected for a non$\mathrm{AD}$ neurodegenerative disorder like frontotemporal lobar degeneration (behavioral or language phenotype) or Parkinsonian disorders $[5,6,8,9]$. This can potentially hamper the interpretation of the tau PET result or lead to suboptimal patient selection for a clinical tau PET scan. A better understanding of factors that contribute to tau PET status could alleviate this concern.

Based on previous literature $[4,7,8,10-23]$ and data availability, we evaluated in this large multicenter study the impact of demographic (age and sex), clinical (Mini-Mental State Examination [MMSE]), genetic (apolipoprotein $[A P O E]$ genotype), and imaging (cortical thickness, white matter hyperintensities [WMH], and amyloid- $\beta[\mathrm{A} \beta]$ status) variables on tau PET status across persons with $\mathrm{AD}$ dementia, mild cognitive impairment (MCI), and non- $\mathrm{AD}$ neurodegenerative disorders, and cognitively unimpaired (CU) individuals.

\section{Material and methods}

\section{Participants}

We included 2338 participants from the Memory Disorder Clinic of Gangnam Severance Hospital (Seoul, South Korea, $n=310)$ and the Swedish BioFINDER $1(n=228)$ and $2(n=$ 719) studies at Lund University (Lund, Sweden), the University of California San Francisco (UCSF) AD
Research Center (San Francisco, USA, $n=201$ ), the PREVENT-AD study ( $n=134)$, the Alzheimer's disease neuroimaging initiative (ADNI, $n=655$ ), and Avid Radiopharmaceuticals studies (A04 [ $n=36]$, A05 [ $n=219]$, A08 $[n=76]$, and the placebo arm of the Eli Lilly solanezumab Expedition-3 study $[n=85])$. Of these participants, $1695(72.5 \%)$ underwent $\left[{ }^{18} \mathrm{~F}\right]$ flortaucipir PET and $643(27.5 \%)\left[{ }^{18} \mathrm{~F}\right] \mathrm{RO} 948$ PET. According to the NIA-AA diagnostic criteria $[24,25]$ and a research framework [26], we only included $\mathrm{A} \beta+\mathrm{AD}$ dementia $(n=430)$ and MCI $(n=381)$ patients (determined using PET and/or CSF, see previous work for details $[5,6])$. A total of 370 participants were diagnosed with a non-AD neurodegenerative disorder according to formal diagnostic criteria [5, 6], including Parkinson's disease $(n=123)$, progressive supranuclear palsy $(n=58)$, dementia with Lewy bodies $(n=51)$, behavioral variant frontotemporal dementia $(n=50)$, corticobasal syndrome $(n=27)$, the semantic $(n=19)$ and non-fluent $(n=17)$ variants of primary progressive aphasia, multiple system atrophy $(n=$ $12)$, and dementia not otherwise specified $(n=14)$. CU individuals $(n=1157)$ performed within normal limits on neuropsychological testing and had no significant neurological or psychiatric illnesses. In addition to tau PET, all participants underwent a medical history and neurological examination, MRI, and neuropsychological testing. Written informed consent was obtained from all participants, and local institutional review boards for human research approved the study. The study was performed in accordance with the ethical standards as laid down in the 1964 Declaration of Helsinki and its later amendments or comparable ethical standards.

\section{Acquisition of PET and MRI data}

PET images were acquired using a Biograph mCT PET/CT scanner (Siemens Medical Solutions) in Seoul [11]; Discovery and Discovery MI PET scanners (GE medical systems) in BioFINDER 1 and 2, respectively [5, 6]; a Biograph 6 Truepoint PET/CT scanner (Siemens Medical Solutions) for UCSF patients [18], a Siemens High-Resolution Research Tomograph (HRRT) scanner in PREVENT-AD [15], and across multiple scanners in the multi-center ADNI [27] and Avid Radiopharmaceuticals [7] cohorts. All PET data were locally reconstructed into $4 \times 5$-min frames for the $80-100$ $\min \left(\left[{ }^{18} \mathrm{~F}\right]\right.$ flortaucipir) and $70-90-\mathrm{min}\left(\left[{ }^{18} \mathrm{~F}\right] \mathrm{RO} 948\right)$ intervals post-injection. MR images were acquired on a 3.0T Discovery 
MR750 scanner (GE medical systems) in Seoul [11], 3.0T Tim Trio or Skyra scanner (Siemens Medical Solutions) in BioFINDER $[5,6]$, a 3.0T Tim Trio or Prisma scanner (Siemens Medical Solutions) at UCSF [18], a 3.0 Tim Trio scanner (Siemens Medical Solutions) in PREVENT-AD [15], and across multiple scanners in the multi-center ADNI [27] and Avid Radiopharmaceuticals [7] cohorts.

\section{T1-weighted MRI processing}

MRI data were centrally processed (at Lund University), using previously reported procedures $[5,6]$. Briefly, cortical reconstruction and volumetric segmentation were performed with the FreeSurfer (v6.0) image analysis pipelines (http://surfer. nmr.mgh.harvard.edu/). The MP-RAGE images underwent correction for intensity homogeneity [28], removal of nonbrain tissue [29], and segmentation into gray matter (GM) and white matter (WM) with intensity gradient and connectivity among voxels [30]. Cortical thickness was measured as the distance from the GM/WM boundary to the corresponding pial surface [31]. Reconstructed data sets were visually inspected for accuracy, and segmentation errors were corrected. We computed "AD-signature" cortical thickness [32] comprising bilateral entorhinal, inferior and middle temporal, and fusiform cortex regions of interest (ROI).

\section{$\left[{ }^{18}\right.$ F]flortaucipir PET processing}

PET images were first re-sampled to obtain the same image size $(128 \times 128 \times 63$ matrix $)$ and voxel dimensions $(2.0 \times 2.0 \times$ $2.0 \mathrm{~mm}$ ) across centers. Next, PET images were centrally processed (at Lund University) using previously reported procedures $[5,6] .\left[{ }^{18} \mathrm{~F}\right]$ Flortaucipir images were motion-corrected using AFNI's 3dvolreg, time-averaged and rigidly co-registered to the skull-stripped MRI scan. Voxelwise standardized uptake value ratio (SUVR) images were created using inferior cerebellar gray matter as the reference region [33]. FreeSurfer (v6.0) parcellation of the T1-weighted MRI scan was applied to the PET data transformed to subjects' native T1 space to extract mean regional SUVR values. In line with our previous work $[5,6]$, we calculated the mean $\left[{ }^{18} \mathrm{~F}\right]$ flortaucipir and $\left[{ }^{18} \mathrm{~F}\right]$ RO948 SUVR in the entorhinal cortex (early tau region), a temporal meta-ROI [32] comprised of a weighted average of entorhinal, amygdala, parahippocampal, fusiform, and inferior and middle temporal ROIs (intermediate tau region), and Braak stage V/VI encompassing widespread neocortical ROIs (late tau region). As tau PET positivity can be established using a variety of methods, we aimed to be consistent with our earlier work and use previously published and validated procedures for both tracers $[5,6]$. Hence, we determined tau PET positivity in each of the aforementioned regions using the mean of elderly $(69.1 \pm$ 9.5 years $) \mathrm{CU}$ subjects $+(2 * \mathrm{SD})$ for $\left[{ }^{18} \mathrm{~F}\right]$ flortaucipir $[6]$ and the mean of young $(30.5 \pm 6.6$ years) $\mathrm{A} \beta$-negative $\mathrm{CU}$ subjects
$+(2.5 * \mathrm{SD})\left[{ }^{18} \mathrm{~F}\right] \mathrm{RO} 948$ [5]. This resulted in cutoffs of 1.39 (entorhinal cortex), 1.34 (temporal meta-ROI), and 1.28 (Braak V-VI) SUVR for $\left[{ }^{18} \mathrm{~F}\right]$ flortaucipir, and 1.48 (entorhinal cortex), 1.36 (temporal meta-ROI), and 1.35 (Braak V-VI) SUVR for $\left[{ }^{18} \mathrm{~F}\right] \mathrm{RO} 948$.

\section{FLAIR MRI processing}

T2-weighted fluid attenuated inversion recovery (FLAIR) images were available for 1660/2338 (71.0\%) participants. We estimated total WMH volumes following a segmentation method described elsewhere [34]. Briefly, this method builds a Bayesian probabilistic data model based on a Gaussian mixture model with an evolving number of components. Due to distribution skewness, data were log-transformed prior to statistical analysis.

\section{Statistical analyses}

To identify factors associated with tau PET positivity, we performed bivariate binary logistic regression models with tau PET status (positive/negative) in the three preselected regions (i.e., entorhinal cortex, temporal meta-ROI, and Braak $\mathrm{V}-\mathrm{VI})$ as dependent variable, and age, sex, $A P O E \varepsilon 4$ status, A $\beta$ status (only in analyses including participants with a nonAD neurodegenerative disorder and CU individuals), MMSE, total WMH volumes (adjusted for intracranial volume), and $\mathrm{AD}$-signature cortical thickness as predictors. Values for ADsignature cortical thickness and total WMH were divided by 10 and 1000 respectively, to obtain odds ratios in a comparable range to the other variables. Additionally, we performed multivariable binary logistic regression models to account for all other predictors in the same model. We excluded total WMH from the multivariable analysis due to a significant proportion (29\%) of missing data. Finally, we conducted a post hoc analyses to estimate at which MMSE score the tau PET scan would be robustly positive in $A \beta$-positive participants with $\mathrm{AD}$ dementia and $\mathrm{MCI}$. Using a non-parametric regression method (i.e., locally estimated scatterplot smoothing [35]), we computed the MMSE score at which the tau PET thresholds diverged from the $95 \%$ confidence interval of the slope reflecting tau PET SUVR vs MMSE scores. Significance level was set at 2 -sided $P<0.05$. We used $\mathrm{R}$ v3.5.2 for the statistical analyses.

\section{Results}

\section{Participants}

The participant characteristics across diagnostic groups are presented in Table 1. Overall, the study participants were $69.4 \pm 10.2$ years old and $53.2 \%$ were males. By definition, 
all $\mathrm{AD}$ dementia and MCI patients were $\mathrm{A} \beta$ positive, and $65.5 \%$ (AD dementia) and 61.8\% (MCI) carried at least one $A P O E \varepsilon 4$ allele. In the non-AD neurodegenerative disorder and $\mathrm{CU}$ group, $29.0 \%$ and $34.3 \%$ were $\mathrm{A} \beta$ positive and $32.5 \%$ and $35.7 \%$ APOE $\varepsilon 4$ positive, respectively. Figure 1 shows the distribution of tau PET SUVR values and the positivity rates across the three ROIs, stratified by tau PET tracer. In the temporal meta-ROI, tau PET positivity was observed in $1.5 \%$ of $\mathrm{A} \beta$-negative $\mathrm{CU}, 2.2 \%$ of $\mathrm{A} \beta$-negative non- $\mathrm{AD}$, $14.7 \%$ of $A \beta$-positive $C U, 27.7 \%$ of $A \beta$-positive non- $A D$, $46.5 \%$ of $\mathrm{A} \beta$-positive $\mathrm{MCI}$, and $88.6 \%$ of $\mathrm{A} \beta$-positive $\mathrm{AD}$ dementia.

\section{Tau PET positivity in amyloid- $\beta+$ AD dementia}

Bivariate and multivariable binary logistic regression models in patients with $\mathrm{A} \beta+\mathrm{AD}$ dementia are presented in Table 2. The multivariable analysis indicated that younger age and lower MMSE scores were associated with higher rates of tau positivity across all ROIs. In addition, lower AD-signature cortical thickness was associated with higher rates of tau positivity in the temporal meta-ROI and the Braak V/VI ROI, and the presence of an $A P O E \& 4$ allele was associated with a higher rate of tau positivity in the temporal meta-ROI only. There were no significant associations between sex and tau PET positivity in both bivariate and multivariable models. WMH volumes were excluded from the multivariable analysis due to missing data, but bivariate models revealed no significant associations between global WMH volumes and tau PET positivity.

\section{Tau PET positivity in amyloid- $\beta+\mathrm{MCI}$}

Bivariate and multivariable binary logistic regression models in patients with $\mathrm{A} \beta+\mathrm{MCI}$ are presented in Table 3. The multivariable analysis indicated that lower MMSE scores and lower $\mathrm{AD}$-signature cortical thickness were associated with higher rates of tau positivity across all ROIs. In addition, presence of an $A P O E \& 4$ allele was associated with higher rates of tau positivity in the entorhinal cortex and temporal meta-ROI, and lower age was associated with a higher rate of tau positivity in the Braak V/VI ROI. There were no significant associations between sex and tau PET positivity in the multivariable models, but male sex was associated with a higher rate of tau positivity in the entorhinal cortex in the bivariate model. WMH volumes were excluded from the multivariable analysis due to missing data. The bivariate analysis indicated a significant association between global WMH volumes and tau PET positivity in the entorhinal cortex, but not in the temporal meta-ROI or the Braak V/VI ROI.

Since a lower MMSE score was consistently associated with higher rates of tau PET positivity across all ROI for both $\mathrm{A} \beta+\mathrm{MCI}$ and $\mathrm{AD}$ dementia, we performed a post hoc analysis to estimate around which MMSE score the tau PET is robustly positive (Fig. 2). This analysis indicated that the $95 \%$ confidence interval did not overlap with the tau PET positivity threshold at an MMSE of 27.6 ( $\left[{ }^{18} \mathrm{~F}\right]$ flortaucipir) or 26.0 $\left(\left[{ }^{18} \mathrm{~F}\right] \mathrm{RO} 048\right)$ for the entorhinal cortex, 29.1 $\left(\left[{ }^{18} \mathrm{~F}\right]\right.$ flortaucipir $)$ or $26.6\left(\left[{ }^{18} \mathrm{~F}\right] \mathrm{RO} 948\right)$ for the temporal meta-ROI, and 22.2 ( $\left[{ }^{18} \mathrm{~F}\right]$ flortaucipir) or $21.9\left(\left[{ }^{18} \mathrm{~F}\right] \mathrm{RO} 948\right)$ for the Braak V/VI ROI.

\section{Tau PET positivity in non-AD neurodegenerative disorders}

Bivariate and multivariable binary logistic regression models in patients with a clinically diagnosed non-AD neurodegenerative disorder are presented in Table 4. The bivariate analysis indicated that most variables (except for sex) were associated with higher rates of tau positivity, especially in the entorhinal

Table 1 Participant characteristics

\begin{tabular}{|c|c|c|c|c|}
\hline & $\mathrm{A} \beta+\mathrm{AD}$ dementia & $A \beta+$ mild cognitive impairment & $\begin{array}{l}\text { Non-AD neurodegenerative } \\
\text { disorders }\end{array}$ & Cognitively unimpaired \\
\hline $\mathrm{N}$ & 430 & 381 & 370 & 1157 \\
\hline Age & $71.5(8.9)$ & $71.6(8.0)$ & $69.4(8.1)$ & $67.9(11.8)$ \\
\hline Sex ( $\%$ male $)$ & 55.6 & 46.5 & 53.5 & 55.3 \\
\hline$A P O E \varepsilon 4$ (\% positive) & 65.5 & 61.8 & 32.5 & 35.7 \\
\hline Amyloid- $\beta$ ( $\%$ positive $)$ & 100 & 100 & 29.0 & 34.3 \\
\hline MMSE & $20.8(4.5)$ & $27.0(2.4)$ & $24.2(5.5)$ & $29.0(1.3)$ \\
\hline$\left[{ }^{18} \mathrm{~F}\right]$ flortaucipir/ $\left[{ }^{18} \mathrm{~F}\right] \mathrm{RO} 948$ (n) & $313 / 117$ & $275 / 106$ & $247 / 123$ & $860 / 297$ \\
\hline AD-signature cortical thickness, mm & $2.41(0.19)$ & $2.60(0.20)$ & $2.57(0.23)$ & $2.74(0.14)$ \\
\hline Global WMH volumes, $\log \mathrm{mm}^{3}$ & $3.71(0.48)$ & $3.68(0.48)$ & $3.62(0.44)$ & $3.48(0.45)$ \\
\hline
\end{tabular}

Data are presented as mean (standard deviation), unless otherwise stated

$A D$ Alzheimer's disease, $A P O E$ apolipoprotein E, MMSE Mini-Mental State Examination, $W M H$ white matter hyperintensities 

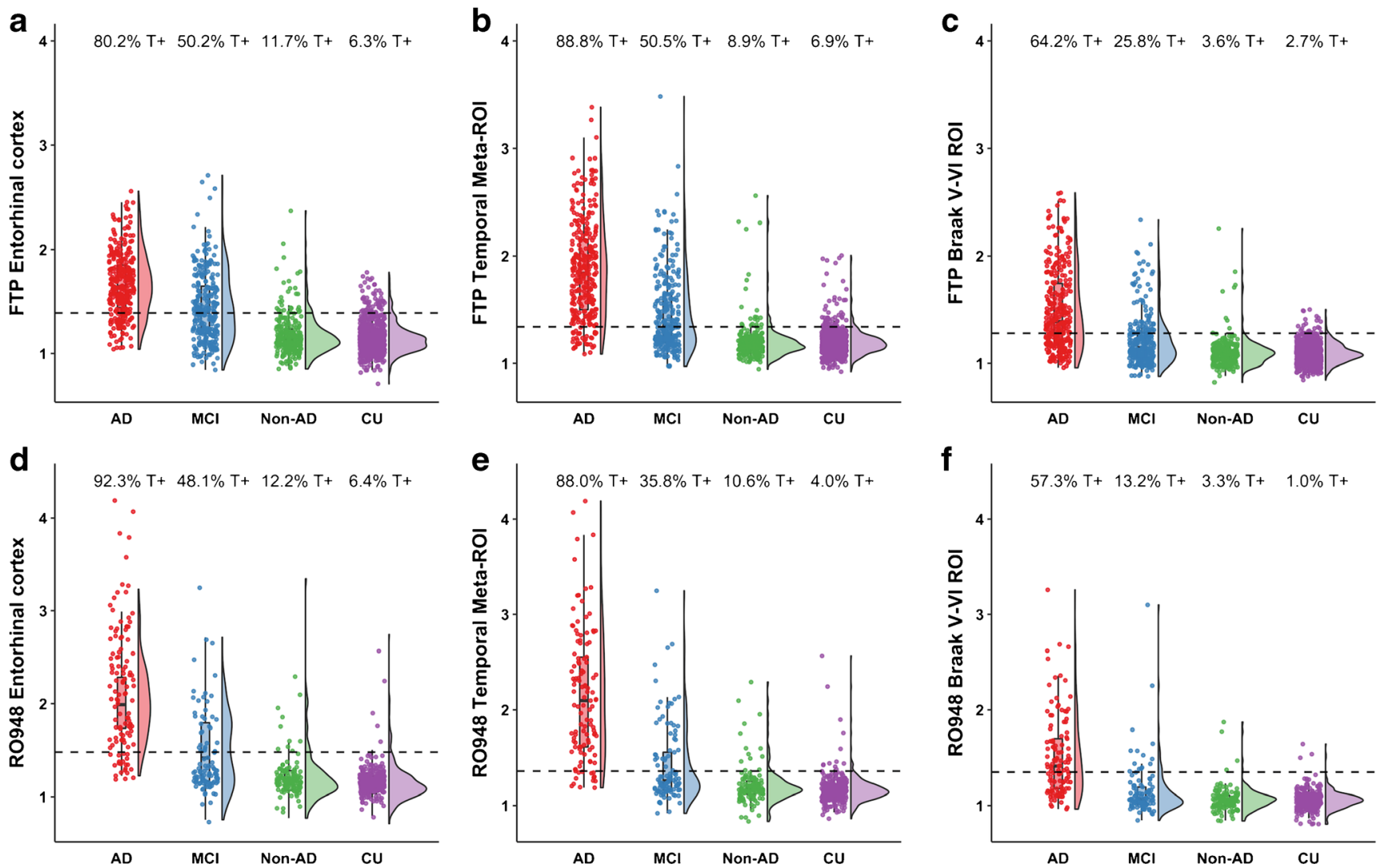

Fig. 1 The $y$-axis represents the SUVR values for $\left[{ }^{18} \mathrm{~F}\right]$ flortaucipir (FTP, panel $\mathbf{a}-\mathbf{c}$ ) and $\left[{ }^{18} \mathrm{~F}\right] \mathrm{RO} 948$ (panel $\mathbf{d}-\mathbf{f}$ ) for the entorhinal cortex (a and d), temporal meta-ROI (b and e), and Braak V/VI ROI for $A \beta+$ Alzheimer's disease (AD) dementia, $A \beta+\mathrm{MCI}$, non-AD

neurodegenerative disorders, and cognitively unimpaired (CU) participants. The percentages on top of each panel represent the proportion of tau PET-positive cases based on previously established cutoffs

cortex and temporal meta-ROI, with $\mathrm{A} \beta$ positivity consistently showing the strongest associations. In the multivariable analysis, only $\mathrm{A} \beta$ positivity and lower MMSE scores remained significantly associated with higher rates of tau positivity in the entorhinal cortex and temporal meta-ROI. There were only 13 tau PET-positive cases in the Braak V-VI ROI (12 of them being $A \beta$ positive), warranting cautious interpretation of these results.

\section{Tau PET positivity in cognitively unimpaired individuals}

Bivariate and multivariable binary logistic regression models in CU participants are presented in Table 5. The bivariate analysis indicated that most variables (except for sex) were associated with higher rates of tau positivity, especially in the entorhinal cortex and temporal meta-ROI, with $A \beta$ positivity consistently showing the strongest associations. In the multivariable analysis with tau PET positivity in the entorhinal cortex as the dependent variable, older age, presence of an $A P O E \& 4$ allele, $\mathrm{A} \beta$ positivity, lower MMSE, and lower ADsignature cortical thickness were associated with higher rates of tau positivity. For the temporal meta-ROI, significant associations were found for older age, male sex, and $\mathrm{A} \beta$ positivity. For the Braak V/VI ROI, significant associations were found for male sex only.

\section{Discussion}

In this large multicenter study, we investigated how demographic (age and sex), clinical (MMSE), genetic (APOE genotype), and imaging/CSF (AD-signature cortical thickness, global WMH volumes, and A $\beta$ status) variables were associated with tau PET status defined using previously established quantitative thresholds for $\left[{ }^{18} \mathrm{~F}\right]$ flortaucipir and $\left[{ }^{18} \mathrm{~F}\right] \mathrm{RO} 948$. In $\mathrm{A} \beta$-positive $\mathrm{AD}$ dementia and $\mathrm{MCI}$, younger age, lower MMSE scores, and lower AD-signature cortical thickness showed the strongest associations with tau PET positivity. In non-AD neurodegenerative disorders and CU participants, the presence of $\mathrm{A} \beta$ was the strongest predictor of a positive tau PET scan. We thus identified several demographic, clinical, and neurobiological factors that are important to explain the variance in tau PET retention observed across the AD pathological continuum, non-AD neurodegenerative disorders, and CU persons. 


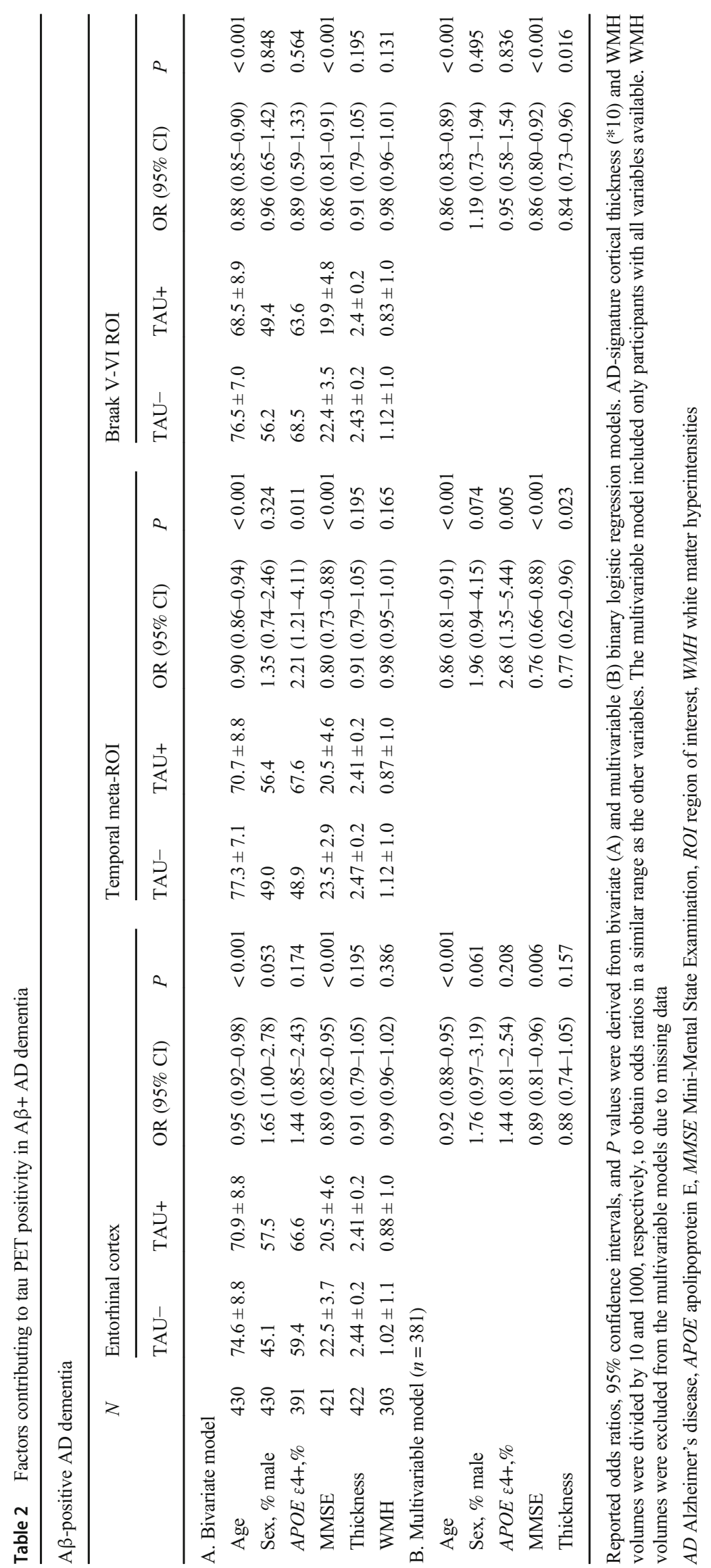




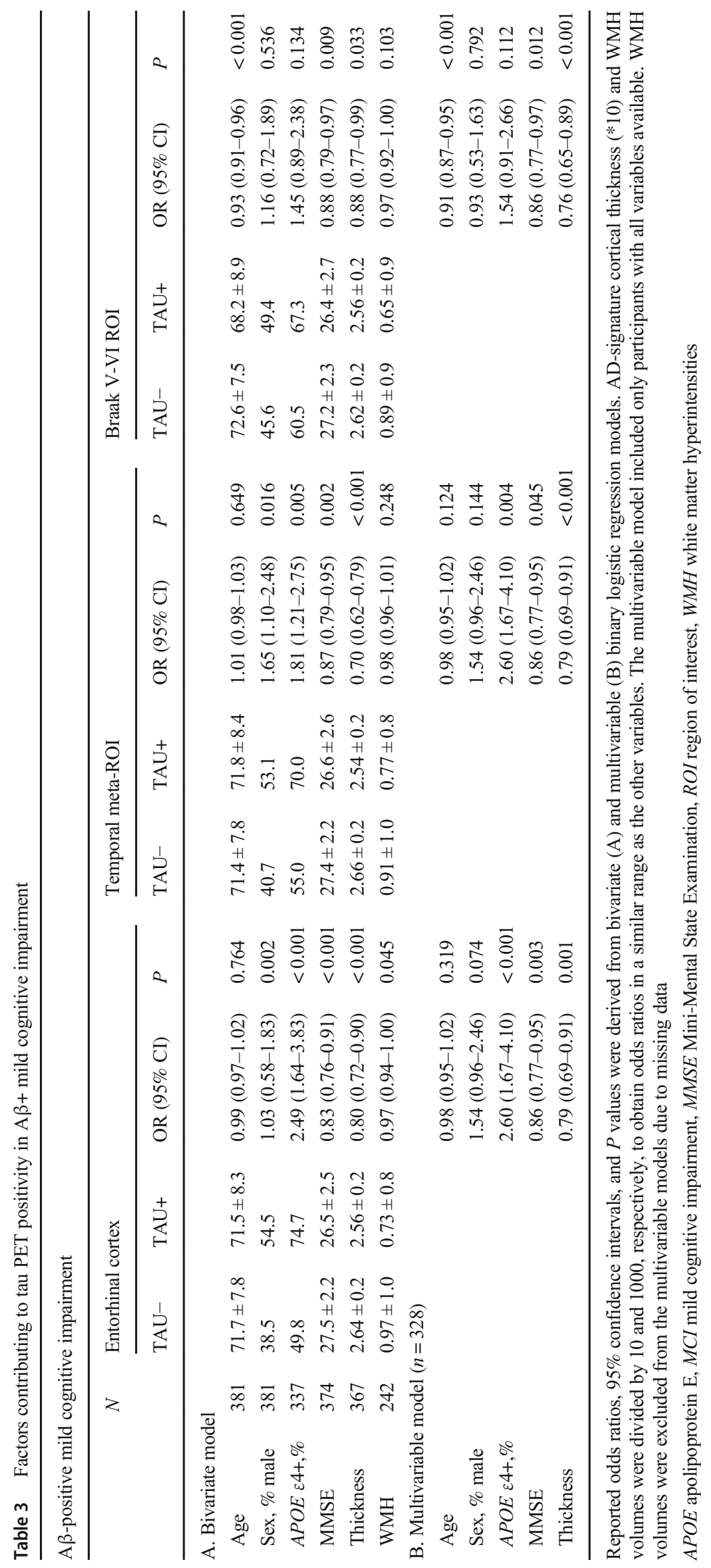



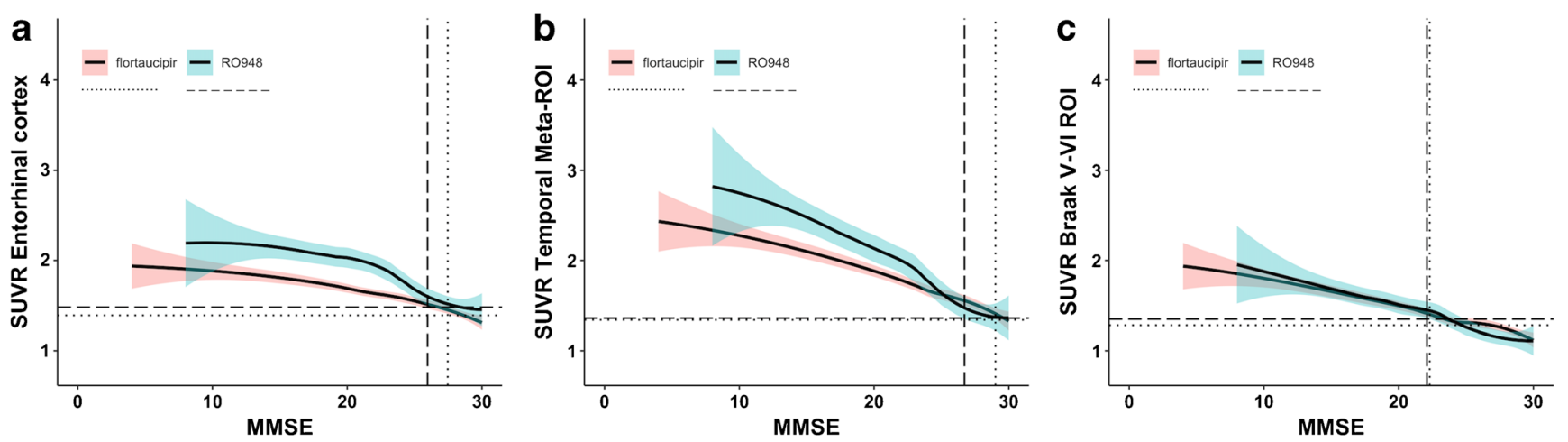

Fig. 2 The graphs show non-linear associations between tau PET uptake and MMSE scores for both $\left[{ }^{18} \mathrm{~F}\right]$ flortaucipir and $\left[{ }^{18} \mathrm{~F}\right] \mathrm{RO} 948$. The horizontal lines (dotted for $\left[{ }^{18} \mathrm{~F}\right]$ flortaucipir and dashed for $\left[{ }^{18} \mathrm{~F}\right] \mathrm{RO} 948$ ) indicate previously established cutoffs for tau PET positivity, while the

Among $A \beta$-positive participants clinically diagnosed with $\mathrm{AD}$ dementia, the main predictors of a negative tau PET scan were older age and a higher MMSE score. At older age, a lower tau burden might be sufficient to produce a dementia syndrome due to the co-occurrence of other age-related pathologies (e.g., TDP-43, $\alpha$-synuclein, or vascular pathology, $[36,37])$ that contribute to cognitive decline (i.e., the "double-hit hypothesis" [38]) or to reduced efficiency of functional repair mechanisms associated with chronological aging that may magnify the cognitive consequences of tau pathology $[16,39]$. Alternatively, in a proportion of participants, their $\mathrm{A} \beta$ positivity may be comorbid to a primary non-AD neurodegenerative disorder [40, 41], which would explain the negative tau PET scan because $\left[{ }^{18} \mathrm{~F}\right]$ flortaucipir and $\left[{ }^{18} \mathrm{~F}\right] \mathrm{RO} 948$ seem to primarily bind to "AD-like" tau pathology and less to the tau aggregates formed in other tauopathies $[42,43]$. A third explanation is the possibility of a selection bias, as older participants with multiple pathologies and other vulnerabilities who additionally have a high tau burden would become too cognitively impaired to participate in clinical research. The greater rates of tau PET-negative status with higher MMSE scores may be explained by the restricted sensitivity of both tau PET tracers $[5,6]$ that requires a significant pathological tau burden in order to cross the quantitative threshold [44], or by an inherent limitation of PET that requires a certain density of the target (Bmax) to allow unambiguous detection. This finding is in line with the drop in tau PET positivity rates in for example the temporal meta-ROI from $\mathrm{AD}$ dementia ( $88 \%$ for both tracers) to MCI (51\% for $\left[{ }^{18} \mathrm{~F}\right]$ flortaucipir and $36 \%$ for $\left.\left[{ }^{18} \mathrm{~F}\right] \mathrm{RO} 948\right)$. More modest associations with tau PET positivity were found for $A P O E$ \&4 status (temporal meta-ROI only) and lower ADsignature cortical thickness (temporal meta-ROI and Braak VVI regions), and sex and global WMH volumes did not impact tau PET status in $\mathrm{A} \beta$-positive $\mathrm{AD}$ dementia.

In $\mathrm{A} \beta$-positive $\mathrm{MCI}$, the main predictors for tau PET positivity were lower MMSE scores and decreased AD-signature cortical thickness. Both could be considered markers of disease progression, and in line with the findings in $\mathrm{AD}$ dementia vertical lines indicate the MMSE score at which the 95\% confidence interval of the slope no longer overlaps with the respective tau PET cutoff for each tracer

and previous literature, there is a tight link between the amount of tau pathology and the level of neurodegeneration and cognitive impairment [11, 19, 22, 45-48]. To a greater degree than in $\mathrm{AD}$ dementia, $A P O E \varepsilon 4$ carriership was associated with a positive tau PET scan in the entorhinal cortex and temporal meta-ROI (but not in the Braak V/VI ROI), which is in accordance with earlier reports of a temporal lobe predilection of $A P O E \varepsilon 4[21,49]$. Furthermore, age played a less prominent role at the MCI stage of $\mathrm{AD}$, as there was only a significant association with (younger) age and tau PET positivity in the Braak V/VI ROI. Sex and global WMH volumes did not impact tau PET status in A $\beta$-positive MCI.

In participants with a non-AD neurodegenerative disorder, positive $A \beta$ status and lower MMSE scores were the only significant predictors of tau PET positivity in the multivariable models. The finding of positive $A \beta$ status can be explained twofold. First, it could be due to a clinical misdiagnosis with $\mathrm{AD}$ as the primary pathological substrate for their dementia syndrome $[41,50]$. This would fit the observation of (lower) MMSE as second predictor of tau PET positivity, as in more advanced disease stages, it may become more difficult to disentangle the symptomatic and radiological features across neurodegenerative disorder and thus increase their clinical and neuroanatomical overlap. Second, $A \beta$ and tau pathology could be present as secondary pathology, whereas the clinical syndrome is driven by non-AD pathologies, as in aging populations where multiple proteinopathies can emerge in tandem [37]. It should be noted that in bivariate models, nearly all variables (except for sex) were associated with tau PET positivity, with the directionality of these associations always in line with $\mathrm{AD}$ risk factors (i.e., older age, $A P O E \varepsilon 4$ positivity, lower MMSE, reduced AD-signature cortical thickness, and elevated global white matter hyperintensity volumes), but these associations did not survive in multivariable models accounting for $\mathrm{A} \beta$ status. Finally, results for Braak V/VI regions should be interpreted with caution, as only 13 participants were tau PET positive in this group. 


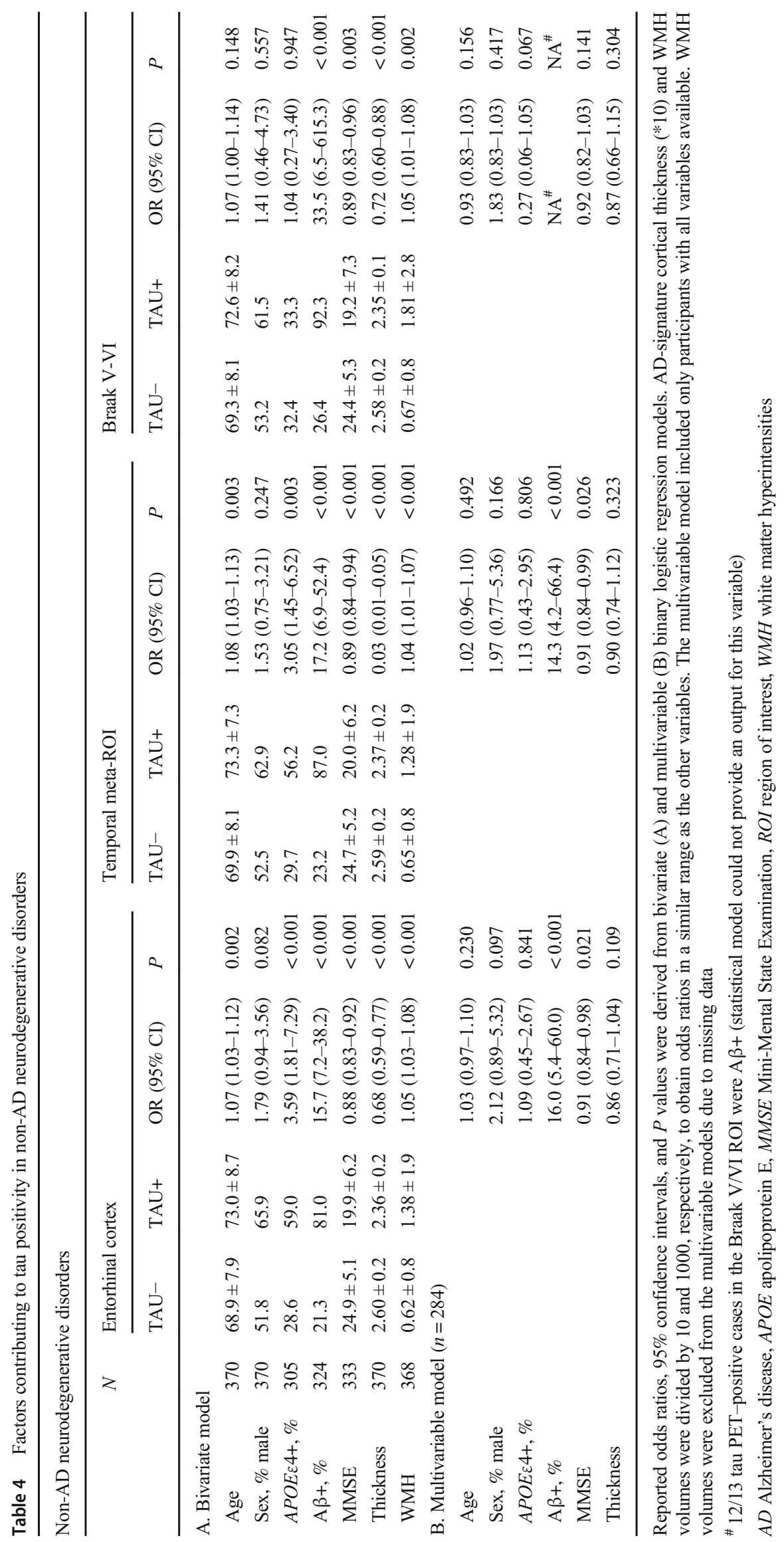




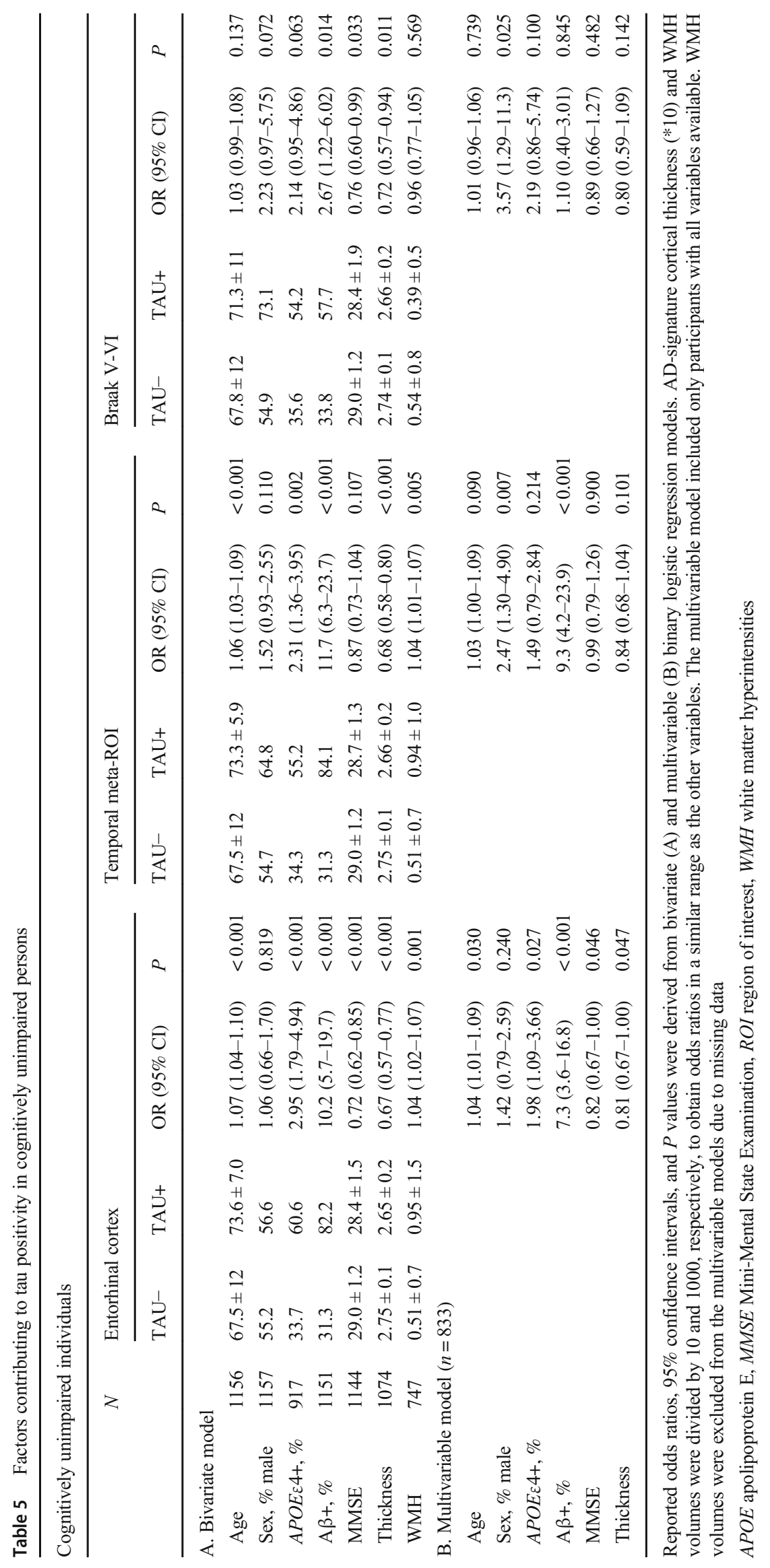


In $\mathrm{CU}$ individuals, positive $\mathrm{A} \beta$ status and older age were the strongest predictors of tau PET positivity in the entorhinal cortex and temporal meta-ROI. This is in line with an extensive literature on the interplay between $A \beta$ and tau pathology in aging populations, suggesting that amyloid- $\beta$ facilitates the neocortical spread of tau pathology [51]. In addition, there is evidence for a (possibly A $\beta$-independent) effect of advancing age on tau accumulation in the medial temporal lobe, which would explain why older age was only associated with tau PET positivity in the entorhinal cortex and temporal meta-ROI, but not in the Braak V-VI ROI. The multivariable model for the Braak V-VI ROI indicated male sex as the only significant predictor of tau PET status, but this finding should be interpreted with caution because this association did not reach significance in the bivariate model and the number of tau PET-positive CU participants in this ROI was limited. Similar to the results for non-AD neurodegenerative disorders, the bivariate models indicated that more "AD-like" characteristics of the predictors (e.g., APOE \&4 positivity, lower MMSE scores, and reduced AD-signature cortical thickness) were associated with tau PET positivity.

The main strengths of this study include the large sample size, the wide variety of clinical diagnoses, and the use of predefined cutoffs for tau PET positivity. There are also several limitations. First, inherent to the retrospective multicenter study design, harmonization between cohorts is complicated. For the imaging data, we attempted to minimize variability by analyzing all data centrally using an identical pipeline, but we acknowledge that differences in data acquisition and pre-processing remain. There likely also exists heterogeneity in the administration of the MMSE and in determining $A \beta$ status using PET or CSF, the former likely being more conservative. Second, due to lack of statistical power to perform the analyses in individual non-AD neurodegenerative disorders, we combined them into a single diagnostic group. It is possible that the study outcomes would be different when focusing on specific non-AD neurodegenerative disorders. Third, the number of CU individuals $(n=26$, $2.3 \%$ ) and participants with non-AD neurodegenerative disorders $(n=13,3.5 \%)$ who were tau PET positive in the Braak V/VI ROI was low. In addition to early (entorhinal cortex) and intermediate (temporal meta-ROI) tau regions, we aimed to also examine a more advanced tau region (i.e., Braak V/VI), but these results should be interpreted with caution. Fourth, we used two different tau PET tracers in this study and defined tau PET positivity using different albeit predefined methods. Based on a previous head-to-head comparison between $\left[{ }^{18} \mathrm{~F}\right]$ flortaucipir and $\left[{ }^{18} \mathrm{~F}\right] \mathrm{RO} 948$ that demonstrated comparable neocortical tracer retention [52], we deemed it appropriate to combine them in the analyses. Fifth, some of the non-AD tauopathies included in this study (e.g., Progressive supranuclear palsy (PSP) or Corticobasal degeneration (CBD)) are characterized by specific isoforms of tau (i.e., 4R in case of PSP and CBD) that are not sufficiently detectable using $\left[{ }^{18} \mathrm{~F}\right]$ flortaucipir or $\left[{ }^{18} \mathrm{~F}\right] \mathrm{RO} 948$ PET $[5,6]$. With "tau PET positivity" in this study, we refer to the detection of "AD-like" tau aggregates, and we acknowledge that tau PET negativity does not exclude the presence of certain tau aggregates typically observed in other tauopathies.

The recent US Food and Drug Administration approval for use of $\left[{ }^{18} \mathrm{~F}\right]$ flortaucipir PET to support the diagnostic process in patients with suspected $\mathrm{AD}$ dementia represents an important step towards application of tau PET in the clinic. The current study identified several demographic, clinical, and neurobiological factors that help explain the variance of tau PET retention observed across the $\mathrm{AD}$ pathological continuum, non- $\mathrm{AD}$ neurodegenerative disorders, and $\mathrm{CU}$ persons. This information might be incorporated into decision trees tailored to optimize patient selection for a clinical tau PET scan and to facilitate the interpretation of tau PET images. Future studies should focus on further development of visual read metrics [44], whether and how a quantitative threshold approach would add to a visual read, how threshold approaches for different tau PET tracers relate to each other and whether a standardized quantification approach would be feasible, and which cutoff in which ROI provides optimal diagnostic performance. This would be especially important to increase the sensitivity of tau PET tracers to detect tau pathology in early disease stages, while maintaining its excellent specificity.

Funding Open access funding provided by Vrije Universiteit Amsterdam. Work at Lund University was supported by the Swedish Research Council, the Knut and Alice Wallenberg foundation, the Marianne and Marcus Wallenberg foundation, the Strategic Research Area MultiPark (Multidisciplinary Research in Parkinson's disease) at Lund University, the Swedish Alzheimer Foundation, the Swedish Brain Foundation, The Medical Faculty at Lund University, Region Skåne, The Bundy Academy, The Parkinson foundation of Sweden, The Parkinson Research Foundation, the Skåne University Hospital Foundation, and the Swedish federal government under the ALF agreement.

CHS is supported by an Alzheimer's Society Junior Fellowship (ASJF-17-011).

Work at UCSF was supported by the following grants: P30AG062422 (BLM, GDR), P01-AG019724 (BLM, GDR), R01AG038791 (GDR), R01-NS050915 (MLGT), K99 AG065501 (RLJ), R01 AG045611 (GDR), Alzheimer's Association AACSF-19-617663 (DSM), Rainwater Charitable Foundation (GDR).

Doses of ${ }^{18} \mathrm{~F}$-flutemetamol injection were sponsored by GE Healthcare in BioFINDER-1. The precursor of ${ }^{18} \mathrm{~F}$-flutemetamol was sponsored by GE Healthcare in BioFINDER-2. The precursor of ${ }^{18} \mathrm{~F}$ flortaucipir was provided by Avid radiopharmaceuticals. The precursor of ${ }^{18} \mathrm{~F}$-RO948 was provided by Roche.

Data collection and sharing for this project was funded by the Alzheimer's Disease Neuroimaging Initiative (ADNI) (National Institutes of Health Grant U01 AG024904) and DOD ADNI (Department of Defense award number W81XWH-12-2-0012). ADNI is funded by the National Institute on Aging and the National Institute of Biomedical Imaging and Bioengineering, and through generous contributions from the following: AbbVie, Alzheimer's Association; Alzheimer's Drug Discovery Foundation; Araclon Biotech; BioClinica, Inc.; Biogen; Bristol-Myers Squibb Company; CereSpir, Inc.; Cogstate; Eisai Inc.; Elan Pharmaceuticals, Inc.; Eli Lilly and Company; EuroImmun; F. Hoffmann-La Roche Ltd. and its affiliated company Genentech, Inc.; Fujirebio; GE Healthcare; IXICO Ltd.; Janssen Alzheimer Immunotherapy Research \& Development, LLC.; Johnson 
\& Johnson Pharmaceutical Research \& Development LLC.; Lumosity; Lundbeck; Merck \& Co., Inc.; Meso Scale Diagnostics, LLC.; NeuroRx Research; Neurotrack Technologies; Novartis Pharmaceuticals Corporation; Pfizer Inc.; Piramal Imaging; Servier; Takeda Pharmaceutical Company; and Transition Therapeutics. The Canadian Institutes of Health Research is providing funds to support ADNI clinical sites in Canada. Private sector contributions are facilitated by the Foundation for the National Institutes of Health (www.fnih.org). The grantee organization is the Northern California Institute for Research and Education, and the study is coordinated by the Alzheimer's Therapeutic Research Institute at the University of Southern California. ADNI data are disseminated by the Laboratory for Neuro Imaging at the University of Southern California.

PREVENT-AD was launched in 2011 as a $\$ 13.5$ million, 7-year publicprivate partnership using funds provided by McGill University and the Fonds de Recherche du Québec - Santé (FRQ-S), an unrestricted research grant from Pfizer Canada, the J.L. Levesque Foundation, the Lemaire Foundation, the Douglas Hospital Research Centre and Foundation, the Government of Canada, and the Canada Fund for Innovation. The PET scans were supported by an Alzheimer's Association Grant, an Alzheimer Society of Canada grant, and two Canada Institutes of Health Research grants (PJT162091 and PJT-148963).

Data availability Anonymized data from BioFINDER will be shared by request from a qualified academic investigator for the sole purpose of replicating procedures and results presented in the article and as long as data transfer is in agreement with EU legislation on the general data protection regulation and decisions by the Ethical Review Board of Sweden and Region Skåne, which should be regulated in a material transfer agreement.

ADNI data can be downloaded from: www.adni.loni.usc.edu.

Most of the PREVENT-AD data can be downloaded from https:// registeredpreventad.loris.ca.

\section{Compliance with ethical standards}

Written informed consent was obtained from all participants, and local institutional review boards for human research approved the study. The study was performed in accordance with the ethical standards as laid down in the 1964 Declaration of Helsinki and its later amendments or comparable ethical standards.

Conflict of interest $\mathrm{OH}$ has acquired research support (for the institution) from Roche, Pfizer, GE Healthcare, Biogen, Eli Lilly, and AVID Radiopharmaceuticals. In the past 2 years, he has received consultancy/ speaker fees (paid to the institution) from Biogen and Roche.

GDR receives research support from NIH, Alz Assoc, American College of Radiology, Avid Radiopharmaceuticals, GE Healthcare, Life Molecular Imaging. In the past 2 years he has received consulting fees from Axon Neurosciences, Eisai, GE Healthcare, Johnson \& Johnson, and Merck. He is an Associate Editor for JAMA Neurology.

ALB receives research support from NIH (R01AG038791, U19AG063911), the Tau Research Consortium, the Association for Frontotemporal Degeneration, and the Bluefield Project to Cure Frontotemporal Dementia. He has served as a consultant for AGTC, Alector, Arkuda, Arvinas, Bioage, Ionis, Lundbeck, Passage BIO, Samumed, Ono, Sangamo, Stealth, Third Rock, Transposon, UCB, and Wave, and received research support from Avid, Eisai, Biogen, and Roche.

MJP and MDD are employees of Avid Radiopharmaceuticals, a wholly owned subsidiary of Eli Lilly and Company, and are minor stockholders in Eli Lilly.

EB and GK are employees of F. Hoffmann-La Roche Ltd.

The other authors report no conflict of interest.

Code availability Code will be provided upon reasonable request.
Open Access This article is licensed under a Creative Commons Attribution 4.0 International License, which permits use, sharing, adaptation, distribution and reproduction in any medium or format, as long as you give appropriate credit to the original author(s) and the source, provide a link to the Creative Commons licence, and indicate if changes were made. The images or other third party material in this article are included in the article's Creative Commons licence, unless indicated otherwise in a credit line to the material. If material is not included in the article's Creative Commons licence and your intended use is not permitted by statutory regulation or exceeds the permitted use, you will need to obtain permission directly from the copyright holder. To view a copy of this licence, visit http://creativecommons.org/licenses/by/4.0/.

\section{References}

1. Selkoe DJ. The molecular pathology of Alzheimer's disease. Neuron. 1991;6:487-98. https://doi.org/10.1016/0896-6273(91) 90052-2.

2. Leuzy A, Chiotis K, Lemoine L, Gillberg PG, Almkvist O, Rodriguez-Vieitez E, et al. Tau PET imaging in neurodegenerative tauopathies-still a challenge. Mol Psychiatry. 2019;24:1112-34. https://doi.org/10.1038/s41380-018-0342-8.

3. US Food \& Drug administration. https://www.fda.gov/news-events/ press-announcements/fda-approves-first-drug-image-tau-pathologypatients-being-evaluated-alzheimers-disease. Accessed 28 May 2020.

4. Jack CR, Wiste HJ, Botha H, Weigand SD, Therneau TM, Knopman DS, et al. The bivariate distribution of amyloid-beta and tau: relationship with established neurocognitive clinical syndromes. Brain. 2019;142:3230-42. https://doi.org/10.1093/brain/ awz268.

5. Leuzy A, Smith R, Ossenkoppele R, Santillo A, Borroni E, Klein G, et al. Diagnostic performance of RO948 F 18 tau positron emission tomography in the differentiation of Alzheimer disease from other neurodegenerative disorders. JAMA Neurol. 2020. https://doi.org/ 10.1001/jamaneurol.2020.0989.

6. Ossenkoppele R, Rabinovici GD, Smith R, Cho H, Scholl M, Strandberg O, et al. Discriminative accuracy of [18F]flortaucipir positron emission tomography for Alzheimer disease vs other neurodegenerative disorders. JAMA. 2018;320:1151-62. https://doi. org/10.1001/jama.2018.12917.

7. Pontecorvo MJ, Devous MD Sr, Navitsky M, Lu M, Salloway S, SchaerfFW, et al. Relationships between flortaucipir PET tau binding and amyloid burden, clinical diagnosis, age and cognition. Brain. 2017;140:748-63. https://doi.org/10.1093/brain/aww334.

8. Bevan-Jones WR, Cope TE, Jones PS, Passamonti L, Hong YT, Fryer TD, et al. [(18)F]AV-1451 binding in vivo mirrors the expected distribution of TDP-43 pathology in the semantic variant of primary progressive aphasia. J Neurol Neurosurg Psychiatry. 2018;89:1032-7. https://doi.org/10.1136/jnnp-2017-316402.

9. Schonhaut DR, McMillan CT, Spina S, Dickerson BC, Siderowf A, Devous MD Sr, et al. (18) F-flortaucipir tau positron emission tomography distinguishes established progressive supranuclear palsy from controls and Parkinson disease: a multicenter study. Ann Neurol. 2017;82:622-34. https://doi.org/10.1002/ana.25060.

10. Buckley RF, Mormino EC, Rabin JS, Hohman TJ, Landau S, Hanseeuw BJ, et al. Sex differences in the Association of Global Amyloid and Regional Tau Deposition Measured by positron emission tomography in clinically normal older adults. JAMA Neurol. 2019;76:542-51. https://doi.org/10.1001/jamaneurol.2018.4693.

11. Cho H, Choi JY, Hwang MS, Lee JH, Kim YJ, Lee HM, et al. Tau PET in Alzheimer disease and mild cognitive impairment. 
Neurology. 2016;87:375-83. https://doi.org/10.1212/WNL. 0000000000002892

12. Graff-Radford J, Arenaza-Urquijo EM, Knopman DS, Schwarz CG, Brown RD, Rabinstein AA, et al. White matter hyperintensities: relationship to amyloid and tau burden. Brain. 2019;142:2483-91. https://doi.org/10.1093/brain/awz162.

13. La Joie R, Visani AV, Baker SL, Brown JA, Bourakova V, Cha J, et al. Prospective longitudinal atrophy in Alzheimer's disease correlates with the intensity and topography of baseline tau-PET. Sci Transl Med. 2020;12. https://doi.org/10.1126/scitranslmed. aau5732.

14. Mattsson N, Ossenkoppele R, Smith R, Strandberg O, Ohlsson T, Jogi J, et al. Greater tau load and reduced cortical thickness in APOE epsilon4-negative Alzheimer's disease: a cohort study. Alzheimers Res Ther. 2018;10:77. https://doi.org/10.1186/ s13195-018-0403-x.

15. McSweeney M, Pichet Binette A, Meyer PF, Gonneaud J, Bedetti $\mathrm{C}$, Ozlen $\mathrm{H}$, et al. Intermediate flortaucipir uptake is associated with Abeta-PET and CSF tau in asymptomatic adults. Neurology. 2020;94:e1190-e200. https://doi.org/10.1212/WNL. 0000000000008905 .

16. Ossenkoppele R, Lyoo CH, Jester-Broms J, Sudre CH, Cho H, Ryu $\mathrm{YH}$, et al. Assessment of demographic, genetic, and imaging variables associated with brain resilience and cognitive resilience to pathological tau in patients with Alzheimer disease. JAMA Neurol. 2020;77:632-42. https://doi.org/10.1001/jamaneurol. 2019.5154 .

17. Ossenkoppele R, Lyoo CH, Sudre CH, van Westen D, Cho H, Ryu $\mathrm{YH}$, et al. Distinct tau PET patterns in atrophy-defined subtypes of Alzheimer's disease. Alzheimers Dement. 2020;16:335-44. https:// doi.org/10.1016/j.jalz.2019.08.201.

18. Ossenkoppele R, Schonhaut DR, Scholl M, Lockhart SN, Ayakta N, Baker SL, et al. Tau PET patterns mirror clinical and neuroanatomical variability in Alzheimer's disease. Brain. 2016;139:155167. https://doi.org/10.1093/brain/aww027.

19. Ossenkoppele R, Smith R, Ohlsson T, Strandberg O, Mattsson N, Insel PS, et al. Associations between tau, Abeta, and cortical thickness with cognition in Alzheimer disease. Neurology. 2019;92: e601-e12. https://doi.org/10.1212/WNL.0000000000006875.

20. Scholl M, Ossenkoppele R, Strandberg O, Palmqvist S, Swedish Bio F, Jogi J, et al. Distinct 18F-AV-1451 tau PET retention patterns in early- and late-onset Alzheimer's disease. Brain. 2017;140: 2286-94. https://doi.org/10.1093/brain/awx171.

21. Therriault J, Benedet AL, Pascoal TA, Mathotaarachchi S, Chamoun M, Savard M, et al. Association of apolipoprotein E epsilon4 with medial temporal tau independent of amyloid-beta. JAMA Neurol. 2020;77:470-9. https://doi.org/10.1001/ jamaneurol.2019.4421.

22. Timmers T, Ossenkoppele R, Wolters EE, Verfaillie SCJ, Visser D, Golla SSV, et al. Associations between quantitative [(18)F]flortaucipir tau PET and atrophy across the Alzheimer's disease spectrum. Alzheimers Res Ther. 2019;11:60. https://doi.org/ 10.1186/s13195-019-0510-3.

23. Whitwell JL, Graff-Radford J, Tosakulwong N, Weigand SD, Machulda MM, Senjem ML, et al. Imaging correlations of tau, amyloid, metabolism, and atrophy in typical and atypical Alzheimer's disease. Alzheimers Dement. 2018;14:1005-14. https://doi.org/10.1016/j.jalz.2018.02.020.

24. Albert MS, DeKosky ST, Dickson D, Dubois B, Feldman HH, Fox $\mathrm{NC}$, et al. The diagnosis of mild cognitive impairment due to Alzheimer's disease: recommendations from the National Institute on Aging-Alzheimer's Association workgroups on diagnostic guidelines for Alzheimer's disease. Alzheimers Dement. 2011;7: 270-9. https://doi.org/10.1016/j.jalz.2011.03.008.

25. McKhann GM, Knopman DS, Chertkow H, Hyman BT, Jack CR $\mathrm{Jr}$, Kawas CH, et al. The diagnosis of dementia due to Alzheimer's disease: recommendations from the National Institute on AgingAlzheimer's Association workgroups on diagnostic guidelines for Alzheimer's disease. Alzheimers Dement. 2011;7:263-9. https:// doi.org/10.1016/j.jalz.2011.03.005.

26. Jack CR Jr, Bennett DA, Blennow K, Carrillo MC, Dunn B, Haeberlein SB, et al. NIA-AA research framework: toward a biological definition of Alzheimer's disease. Alzheimers Dement. 2018;14:535-62. https://doi.org/10.1016/j.jalz.2018.02.018.

27. Weiner MW, Veitch DP. Introduction to special issue: overview of Alzheimer's disease neuroimaging initiative. Alzheimers Dement. 2015;11:730-3. https://doi.org/10.1016/j.jalz.2015.05.007.

28. Sled JGZA, Evans AC. A nonparametric method for automatic correction of intensity nonuniformity in MRI data. IEEE Trans Med Imaging. 1998;17:10.

29. Segonne F, Dale AM, Busa E, Glessner M, Salat D, Hahn HK, et al. A hybrid approach to the skull stripping problem in MRI. Neuroimage. 2004;22:1060-75. https://doi.org/10.1016/j. neuroimage.2004.03.032.

30. Fischl B, Salat DH, Busa E, Albert M, Dieterich M, Haselgrove C, et al. Whole brain segmentation: automated labeling of neuroanatomical structures in the human brain. Neuron. 2002;33:341-55. https://doi.org/10.1016/s0896-6273(02)00569-x.

31. Fischl B, Dale AM. Measuring the thickness of the human cerebral cortex from magnetic resonance images. Proc Natl Acad Sci U S A. 2000;97:11050-5. https://doi.org/10.1073/pnas.200033797.

32. Jack CR Jr, Wiste HJ, Weigand SD, Therneau TM, Lowe VJ, Knopman DS, et al. Defining imaging biomarker cut points for brain aging and Alzheimer's disease. Alzheimers Dement. 2017;13:205-16. https://doi.org/10.1016/j.jalz.2016.08.005.

33. Maass A, Landau S, Baker SL, Horng A, Lockhart SN, La Joie R, et al. Comparison of multiple tau-PET measures as biomarkers in aging and Alzheimer's disease. Neuroimage. 2017;157:448-63. https://doi.org/10.1016/j.neuroimage.2017.05.058.

34. Sudre CH, Cardoso MJ, Bouvy WH, Biessels GJ, Barnes J, Ourselin S. Bayesian model selection for pathological neuroimaging data applied to white matter lesion segmentation. IEEE Trans Med Imaging. 2015;34:2079-102. https://doi.org/10.1109/TMI. 2015.2419072.

35. Cleveland WS. Robust locally weighted regression and smoothing scatterplots. J Am Stat Assoc. 1979;74:829-36. https://doi.org/10. $2307 / 2286407$.

36. Nelson PT, Dickson DW, Trojanowski JQ, Jack CR, Boyle PA, Arfanakis K, et al. Limbic-predominant age-related TDP-43 encephalopathy (LATE): consensus working group report. Brain. 2019;142:1503-27. https://doi.org/10.1093/brain/awz099.

37. Karanth S, Nelson PT, Katsumata Y, Kryscio RJ, Schmitt FA, Fardo DW, et al. Prevalence and clinical phenotype of quadruple misfolded proteins in older adults. JAMA Neurol. 2020. https://doi. org/10.1001/jamaneurol.2020.1741.

38. Hardy J. The amyloid hypothesis for Alzheimer's disease: a critical reappraisal. J Neurochem. 2009;110:1129-34. https://doi.org/10. 1111/j.1471-4159.2009.06181.x.

39. Kirkwood TB. Understanding the odd science of aging. Cell. 2005;120:437-47. https://doi.org/10.1016/j.cell.2005.01.027.

40. Jellinger KA, Attems J. Prevalence of dementia disorders in the oldest-old: an autopsy study. Acta Neuropathol. 2010;119:42133. https://doi.org/10.1007/s00401-010-0654-5.

41. Ossenkoppele R, Jansen WJ, Rabinovici GD, Knol DL, van der Flier WM, van Berckel BN, et al. Prevalence of amyloid PET positivity in dementia syndromes: a meta-analysis. JAMA. 2015;313: 1939-49. https://doi.org/10.1001/jama.2015.4669.

42. Honer M, Gobbi L, Knust H, Kuwabara H, Muri D, Koerner M, et al. Preclinical evaluation of (18)F-RO6958948, (11)CRO6931643, and (11)C-RO6924963 as novel PET radiotracers for imaging tau aggregates in Alzheimer disease. J Nucl Med. 2018;59:675-81. https://doi.org/10.2967/jnumed.117.196741. 
43. Marquie M, Normandin MD, Vanderburg CR, Costantino IM, Bien EA, Rycyna LG, et al. Validating novel tau positron emission tomography tracer [F-18]-AV-1451 (T807) on postmortem brain tissue. Ann Neurol. 2015;78:787-800. https://doi.org/10.1002/ana. 24517.

44. Fleisher AS, Pontecorvo MJ, Devous MD Sr, Lu M, Arora AK, Truocchio SP, et al. Positron emission tomography imaging with $[18 \mathrm{~F}]$ flortaucipir and postmortem assessment of Alzheimer disease neuropathologic changes. JAMA Neurol. 2020. https://doi.org/10. 1001/jamaneurol.2020.0528.

45. Bejanin A, Schonhaut DR, La Joie R, Kramer JH, Baker SL, Sosa $\mathrm{N}$, et al. Tau pathology and neurodegeneration contribute to cognitive impairment in Alzheimer's disease. Brain. 2017;140:3286300. https://doi.org/10.1093/brain/awx243.

46. Gordon BA, Blazey TM, Christensen J, Dincer A, Flores S, Keefe $\mathrm{S}$, et al. Tau PET in autosomal dominant Alzheimer's disease: relationship with cognition, dementia and other biomarkers. Brain. 2019;142:1063-76. https://doi.org/10.1093/brain/awz019.

47. Scott MR, Hampton OL, Buckley RF, Chhatwal JP, Hanseeuw BJ, Jacobs HI, et al. Inferior temporal tau is associated with accelerated prospective cortical thinning in clinically normal older adults. Neuroimage. 2020;220:116991. https://doi.org/10.1016/j. neuroimage.2020.116991.

48. Jack CR Jr, Wiste HJ, Therneau TM, Weigand SD, Knopman DS, Mielke MM, et al. Associations of amyloid, tau, and neurodegeneration biomarker profiles with rates of memory decline among individuals without dementia. JAMA. 2019;321:2316-25. https://doi.org/10.1001/jama.2019.7437.

49. Ramanan VK, Castillo AM, Knopman DS, Graff-Radford J, Lowe VJ, Petersen RC, et al. Association of apolipoprotein E varepsilon4, educational level, and sex with tau deposition and tau-mediated metabolic dysfunction in older adults. JAMA Netw Open. 2019;2: e1913909. https://doi.org/10.1001/jamanetworkopen.2019.13909.

50. Beach TG, Monsell SE, Phillips LE, Kukull W. Accuracy of the clinical diagnosis of Alzheimer disease at National Institute on Aging Alzheimer Disease Centers, 2005-2010. J Neuropathol Exp Neurol. 2012;71:266-73. https://doi.org/10.1097/NEN. 0b013e31824b211b.

51. Busche MA, Hyman BT. Synergy between amyloid-beta and tau in Alzheimer's disease. Nat Neurosci. 2020. https://doi.org/10.1038/ s41593-020-0687-6.

52. Smith R, Scholl M, Leuzy A, Jogi J, Ohlsson T, Strandberg O, et al. Head-to-head comparison of tau positron emission tomography tracers [(18)F]flortaucipir and [(18)F]RO948. Eur J Nucl Med Mol Imaging. 2020;47:342-54. https://doi.org/10.1007/s00259019-04496-0.

Publisher's note Springer Nature remains neutral with regard to jurisdictional claims in published maps and institutional affiliations.

\section{Affiliations}

Rik Ossenkoppele ${ }^{1,2}$ (D) Antoine Leuzy ${ }^{1} \cdot$ Hanna Cho $^{3} \cdot$ Carole H. Sudre $^{4,5,6} \cdot$ Olof Strandberg $^{1} \cdot$ Ruben Smith $^{1}$. Sebastian Palmqvist ${ }^{1}$ • Niklas Mattsson-Carlgren ${ }^{1,7,8} \cdot$ Tomas Olsson $^{9} \cdot$ Jonas Jögi $^{10} \cdot$ Erik Stormrud $^{1,11}$. Young Hoon Ryu ${ }^{12}$. Jae Yong Choi ${ }^{12,13} \cdot$ Adam L. Boxer $^{14} \cdot$ Maria L. Gorno-Tempini ${ }^{14}$. Bruce L. Miller ${ }^{14}$. David Soleimani-Meigooni ${ }^{14} \cdot$ Leonardo laccarino $^{14} \cdot$ Renaud La Joie $^{14}$. Edilio Borroni ${ }^{15} \cdot$ Gregory Klein $^{15}$.

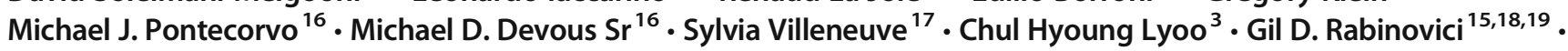
Oskar Hansson ${ }^{1,11}$ • for the Alzheimer's Disease Neuroimaging Initiative • for the PREVENT-AD research group

1 Clinical Memory Research Unit, Lund University, Lund, Sweden

2 Alzheimer Center Amsterdam, Department of Neurology, Amsterdam Neuroscience, Amsterdam UMC, VU University Medical Center, Vrije Universiteit Amsterdam,

Amsterdam, The Netherlands

3 Department of Neurology, Gangnam Severance Hospital, Yonsei University College of Medicine, Seoul, South Korea

4 School of Biomedical Engineering and Imaging Sciences, King's College London, London, UK

5 Dementia Research Centre, Department of Neurodegenerative Disease, UCL Institute of Neurology, London, UK

6 Centre for Medical Image Computing, Department of Medical Physics, University College London, London, UK

7 Department of Neurology, Skåne University Hospital, Lund, Sweden

8 Wallenberg Centre for Molecular Medicine, Lund University, Lund, Sweden

9 Department of Radiation Physics, Skåne University Hospital, Lund, Sweden
10 Department of Clinical Physiology and Nuclear Medicine, Skåne University Hospital, Lund, Sweden

11 Memory Clinic, Skåne University Hospital, Malmö, Sweden

12 Department of Nuclear Medicine, Gangnam Severance Hospital, Yonsei University College of Medicine, Seoul, South Korea

13 Division of applied RI, Korea Institute Radiological and Medical Sciences, Seoul, South Korea

14 Department of Neurology, Memory and Aging Center, University of California San Francisco, San Francisco, USA

15 F. Hoffmann-La Roche Ltd, Basel, Switzerland

16 Avid Radiopharmaceuticals, Philadelphia, PA, USA

17 Departments of Psychiatry and Neurology \& Neurosurgery, Douglas Mental Health University Institute, McGill University, Montreal, Quebec, Canada

18 Department of Radiology and Biomedical Imaging, University of California San Francisco, San Francisco, USA

19 Molecular Biophysics and Integrated Bioimaging Division, Lawrence Berkeley National Laboratory, Berkeley, CA, USA 